\title{
Study the Effects and Compensation of Polarization Mode Dispersion (PMD) at Different Bit Rates
}

\author{
Kapil Kashyap ${ }^{1}$ and Dr. Hardeep Singh $^{2}$ \\ I (ECE Deptt, Thapar University, Patiala, Punjab) INDIA \\ ${ }_{2}^{2}$ (ECE Deptt, Thapar University, Patiala, Punjab) INDIA
}

\begin{abstract}
This paper deals with the effects and compensation of Polarization Mode Dispersion (PMD), when we increase the data rates. Unlike the chromatic dispersion phenomenon, the PMD is found to be a time varying and an unstable phenomenon. Thus compensation of PMD is required since it distorts the signal and broadens the pulse in a statistical manner. In this paper we analyze the PMD effects and compensate the effect of PMD using the OPTSIM simulation for 2.5, 5 and 10 Giga bits per second $(\mathrm{Gb} / \mathrm{s})$ transmission systems. These effects will be analysed for pre and post compensation. Here we will see the output through the eye opening, eye closing, eye diagram and the results have been simulated and analyzed.
\end{abstract}

\section{INTRODUCTION}

The different compensation methods can be divided into different categories, depending on weather the compensation is performed electrically [1,2], optoelectronically [3] or optically or weather the compensation is done before or after compensation (pre or post compensation). A straight forward method to reduce the PMD effects is to reduce the PMD effects optically is to launch the signal into PSP $[4,5]$. Figure 1.1 defined a first order compensation as it enables the compensation to first order only. Optical post compensation is currently an intense research field where both first order and higher order compensation have been suggested. The main problem must be focused upon how the PMD induced distortion should be compensated. Here in the above figures we show first order pre and post compensation. In the post compensation technique we can make it of any order. The benefits of applying PMD compensation is also can also be treated analytically. The results and theory describe the expected pulse broadening for random and PSP oriented input polarizations, as well as the benefit of using an optical post compensation technique. The theory also includes an arbitrarily variable one stage compensator which can partially compensate for higher order PMD. In the above set-ups we have a transmitter section, a channel and a receiver section. We can transmit the signal from the transmitter section. Here, we have Laser source and a data source and an amplitude modulator. The bit rate can be send through the data source and at the amplitude modulator the input signal can be modulated. Then the signal can be passed through the fiber channel. In figure 1.1 which is a pre- compensation technique we have an PMD emulator which act as a PMD compensator before the transmission through the fiber whereas in figure 1.2 which is a post- compensation technique we use PMD emulator after transmission through the fiber. In figures 1.1 and figure 1.2 we use first order compensation technique.

\section{A Optical pre compensation}

\section{THEORY}

It is simple technique to launch the light into the PSP to reduce the PMD effects. However, in practice the feedback signal can be connected back to the transmitter, which makes the compensation inherently slow. This method is obtained by the aligning the input SOP to one of the PSPs. This method can be applied in the installed links [6] as we know that PSPs are obtained in the stokes space whereas, in Jones space, which is more convenient in numerical simulations, the output PSPs are given by the eigen vectors. The block diagram for pre compensation is shown below that how the compensation is done before transmission.

\section{SIMULATION SETUP FOR PRE COMPENSATION}

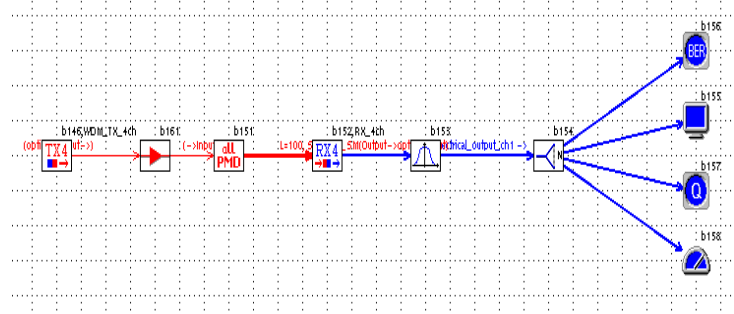

Fig 1.1: OPTSIM layout for optical pre compensation. 
The layout shown in Fig. 4 has been implemented by using the software called OPTSIM which is one of the costliest simulation software's, exclusively meant for the optical communication purposes. It is specially designed, supported and marketed worldwide by RSOFT Design Group. It is also focused entirely on productivity tools for telecommunication and data communication engineering. The components in the layout is for four channels of which we have shown the output of the fourth channel at the output. The data source used here is a Pseudo Random Binary Sequence (PRBS) generator. The period of the waveform, duty cycle, amplitude levels and data rates can be set in this generator. The Direct modulator laser component normally simulates a simplified continuous wave laser and a modulator component, which generates a continuous wave of constant amplitude and modulates the signal. Generally a fiber is after all a transmission medium which should bring the same status of the signal (both if the time domain as well as in frequency domain) at the input and output. At the transmitter end the bit rate is firstly $2.5 \mathrm{~Gb} / \mathrm{s}$, than $5 \mathrm{~Gb} / \mathrm{s}$ and than $10 \mathrm{~Gb} / \mathrm{s}$, the center frequency is 193.55. The fiber used in this experiment is of length $100 \mathrm{~km}$. The attenuation, dispersions such as chromatic and PMD dispersions, Raman effects, SPM, birefringence effects can be set in the fiber, as parameters. This fiber is normally a complex structure and all the effects such as attenuation are caused only because of the fiber nonlinearity. So handling of this component is very important. Generally fiber used here will be the single mode fiber after the simulation, the broadened and compensated pulses can be viewed at different bit rates with the help of signal BER estimator, Q estimator and electrical power meter which are shown below. The graph for the different bit rates are shown below.

\section{B Optical post compensation}

The PMD induced distortion can be compensated for after transmission by introducing a birefringent element which cancels the DGD at the carrier frequency. This compensation element can consists of a number of sections which implies that the scheme has a certain number of control parameters are degree of freedom (DOF). The higher the number of degree of freedom the more flexible the system becomes, enabling compensation for various DGD values and to some extent also for higher order PMD on the other hand, a more complicated control system is required this slows down the response and reduces the likelihood of maintaining the optimized state of the compensating system. The block diagram for optical post compensation after transmission is shown below.

\section{SIMULATION SETUP FOR POST COMPENSATION}

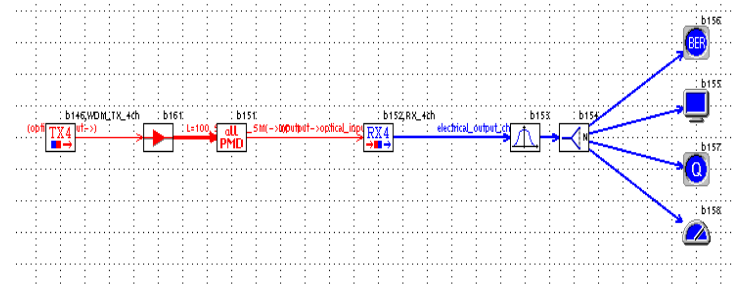

Fig 1.2: OPTSIM layout for optical post compensation.

The layout shown in Fig. 5 has been implemented by using the software called OPTSIM which is one of the costliest simulation softwares, exclusively meant for the optical communication purposes. It is specially designed, supported and marketed worldwide by RSOFT Design Group. It is also focused entirely on productivity tools for telecommunication and data communication engineering. The components in the layout is for four channels of which we have shown the output of the fourth channel at the output. The data source used here is a Pseudo Random Binary Sequence (PRBS) generator. The period of the waveform, duty cycle, amplitude levels and data rates can be set in this generator. The Direct modulator laser component normally simulates a simplified continuous wave laser and a modulator component, which generates a continuous wave of constant amplitude and modulates the signal. Generally a fiber is after all a transmission medium which should bring the same status of the signal (both if the time domain as well as in frequency domain) at the input and output. At the transmitter end the bit rate is firstly $2.5 \mathrm{~Gb} / \mathrm{s}$, than $5 \mathrm{~Gb} / \mathrm{s}$ and than $10 \mathrm{~Gb} / \mathrm{s}$, the center frequency is 193.55. The fiber used in this experiment is of length $100 \mathrm{~km}$. The attenuation, dispersions such as chromatic and PMD dispersions, Raman effects, SPM, birefringence effects can be set in the fiber, as parameters. This fiber is normally a complex structure and all the effects such as attenuation are caused only because of the fiber nonlinearity. So handling of this component is very important. Generally fiber used here will be the single mode fiber after the simulation, the broadened and compensated pulses can be viewed at different bit rates with the help of signal BER estimator, Q estimator and electrical power meter which are shown below. The graph for the different bit rates are shown below. 


\section{RESULTS FOR PRE COMPENSAT-ION}

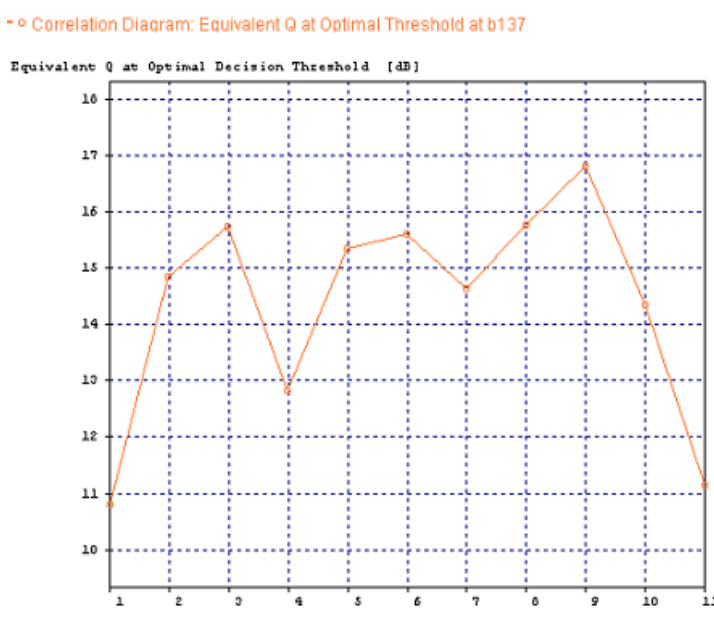

(a)

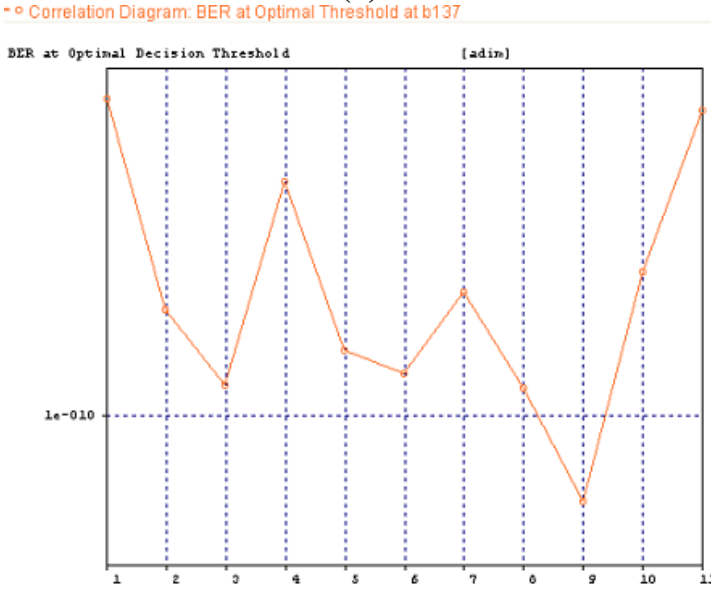

(b)

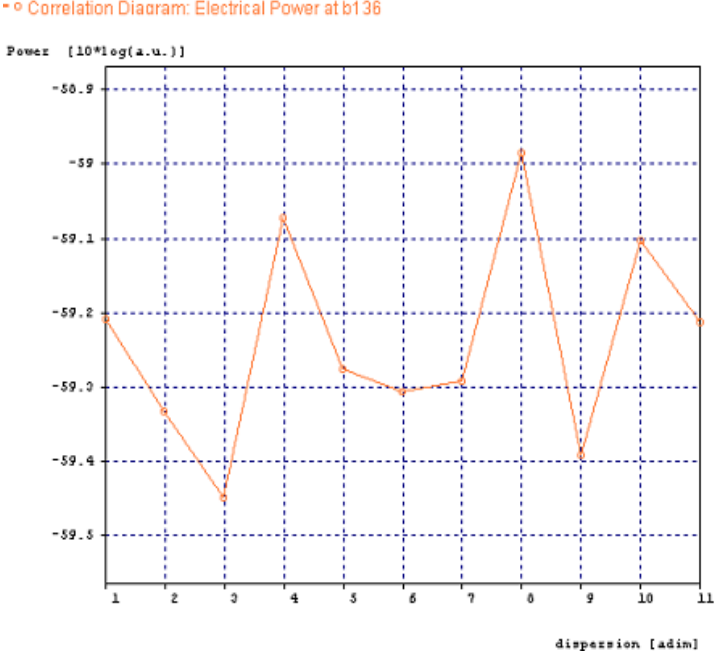

(c)

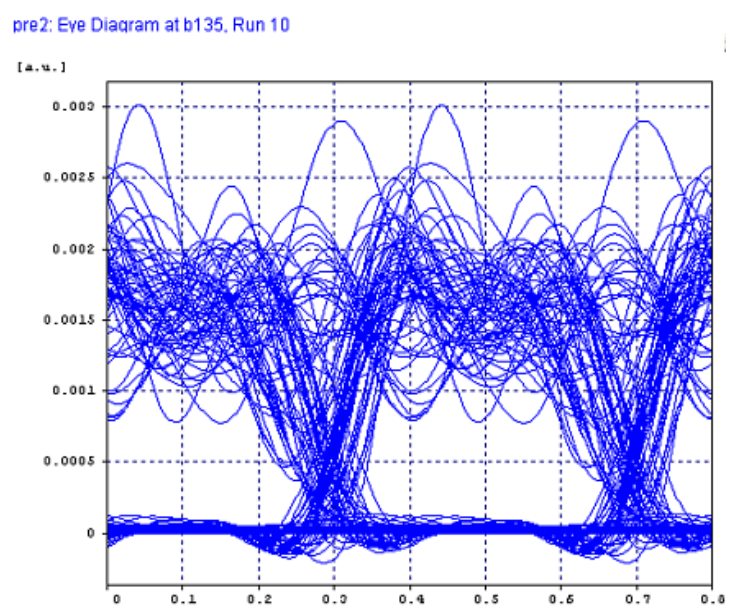

(d)

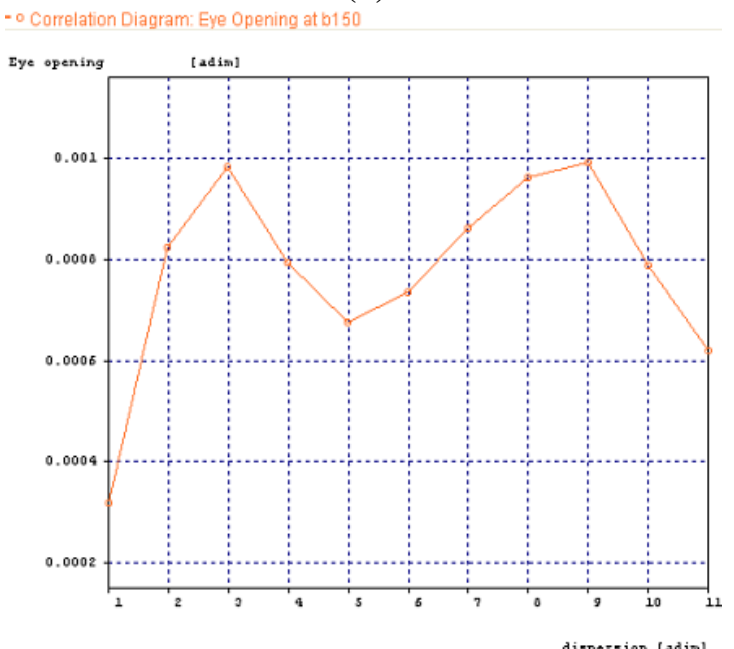

(e)

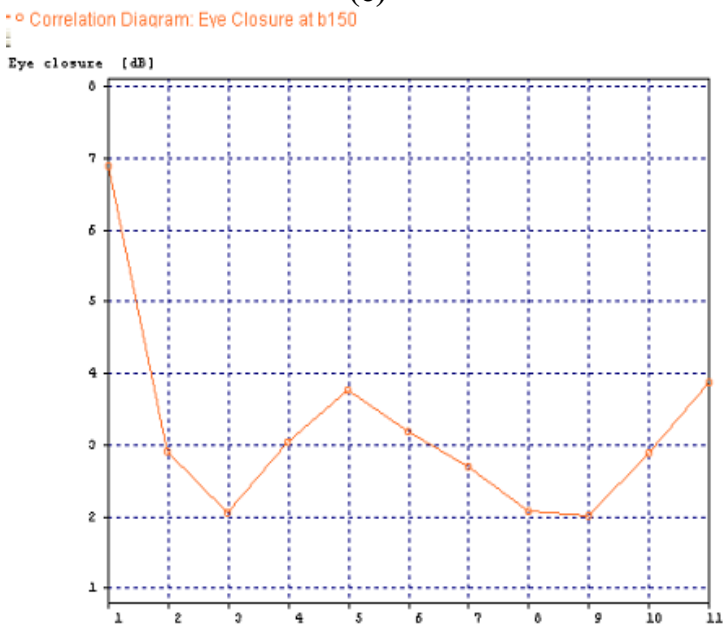

(f)

Figure 1.3: For channel 1 at $2.5 \mathrm{~Gb} / \mathrm{s}$ for NRZ raised cosine (a) PMD verses equivalent $Q$ factor (b) PMD verses BER (c) the PMD verses electrical power (d) eye diagram at $10 \mathrm{ps} / \sqrt{\mathrm{km}}$ (e) PMD verses eye opening (f) PMD verses eye closure. 
The figure 1.4 shows the Q value, BER, electrical power, eye opening, eye closure and eye diagrams at the corresponding values of PMD. All these figures are for the channel one of the transmitter section at $5 \mathrm{~Gb} / \mathrm{s}$.

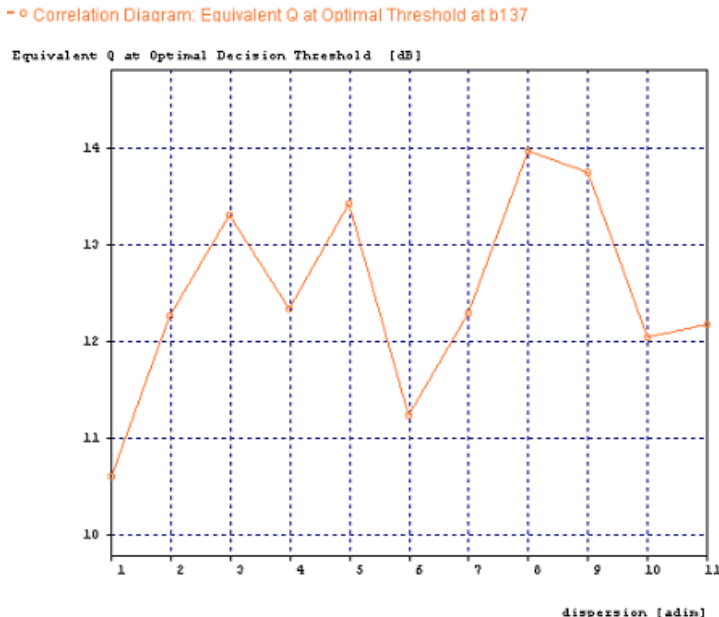

(a)

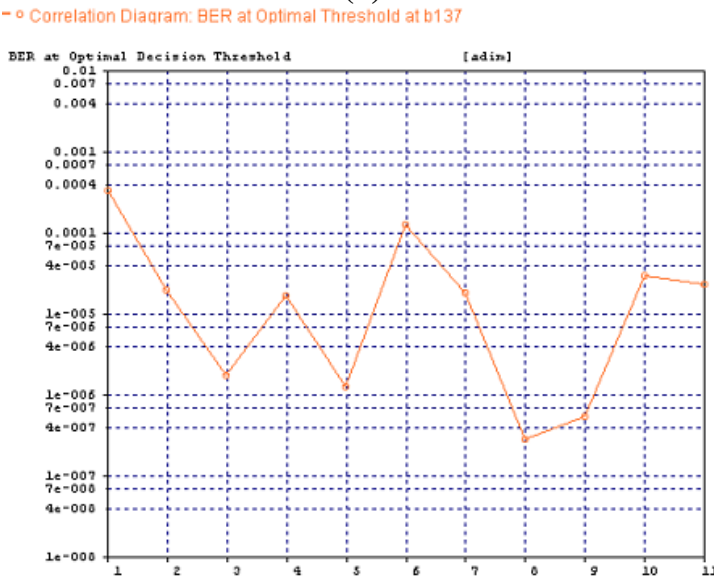

(b)

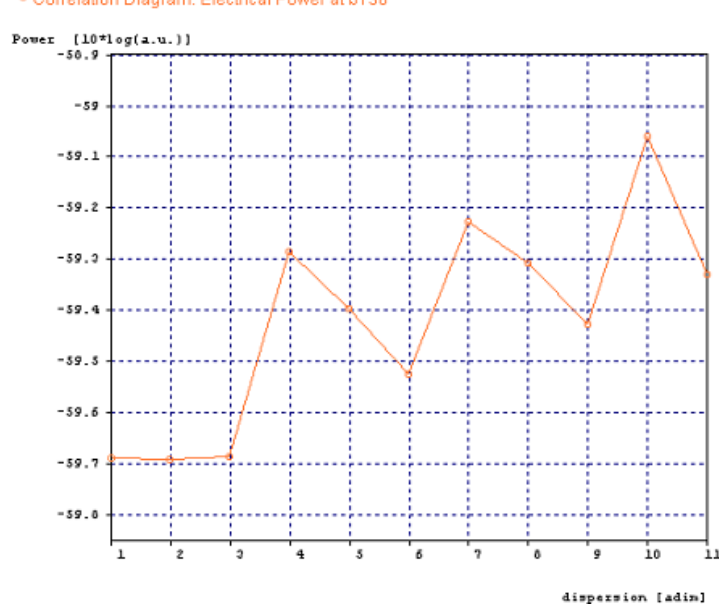

(c)

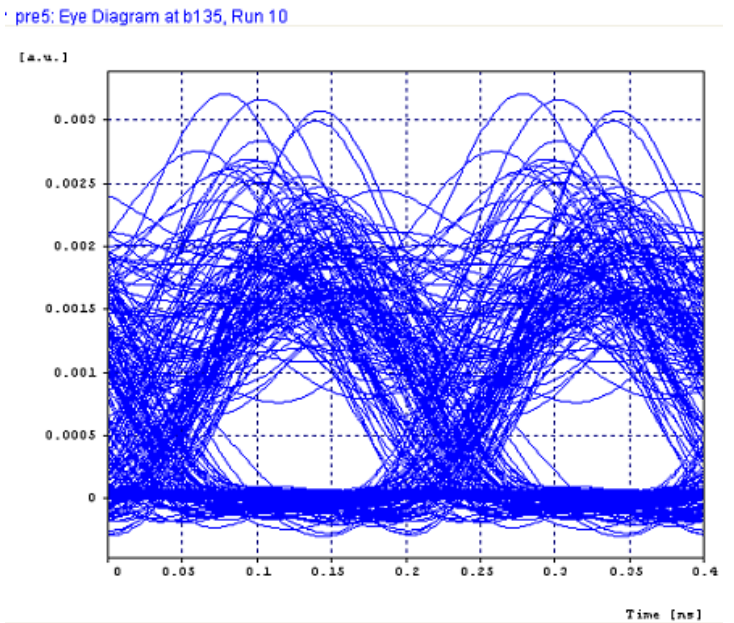

(d)

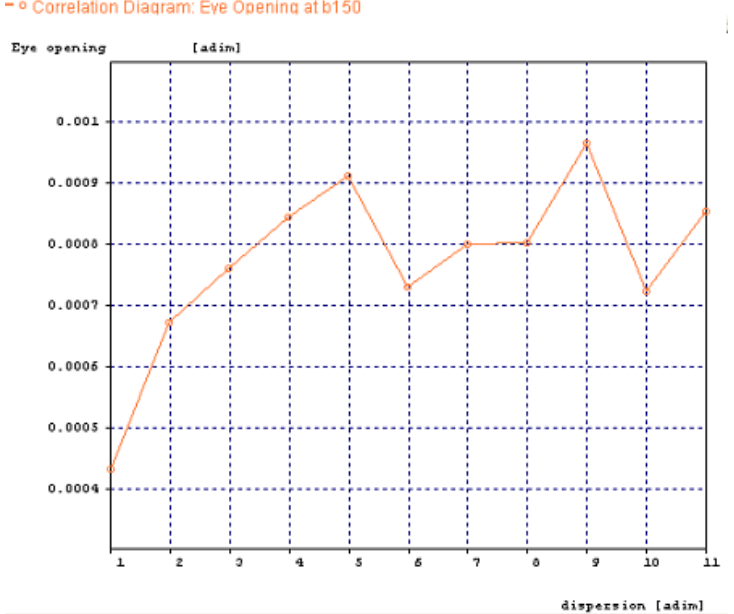

(e)

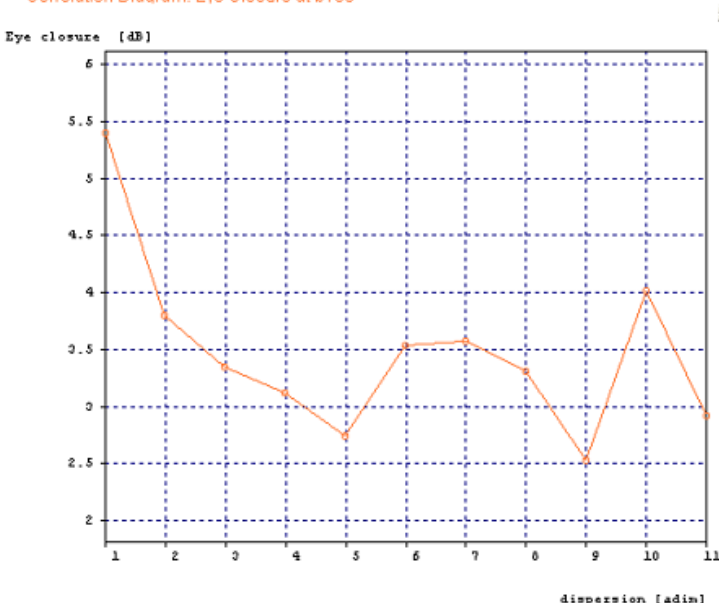

(f)

Figure 1.4: For channel 1 at $5 \mathrm{~Gb} / \mathrm{s}$ for NRZ raised cosine (a) PMD verses equivalent $Q$ factor (b) PMD verses BER (c) the PMD verses electrical power (d) eye diagram at $10 \mathrm{ps} / \sqrt{\mathrm{km}}$ (e) PMD verses eye opening (f) PMD verses eye closure. 
The figure 1.5 shows the Q value, BER, electrical power, eye opening, eye closure and eye diagrams at the corresponding values of PMD. All these figures are for the channel one of the transmitter section at $10 \mathrm{~Gb} / \mathrm{s}$.

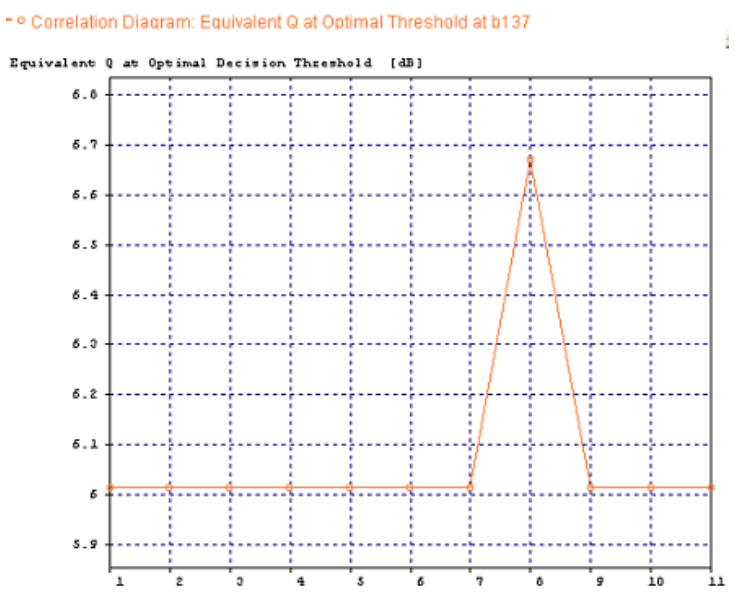

(a)

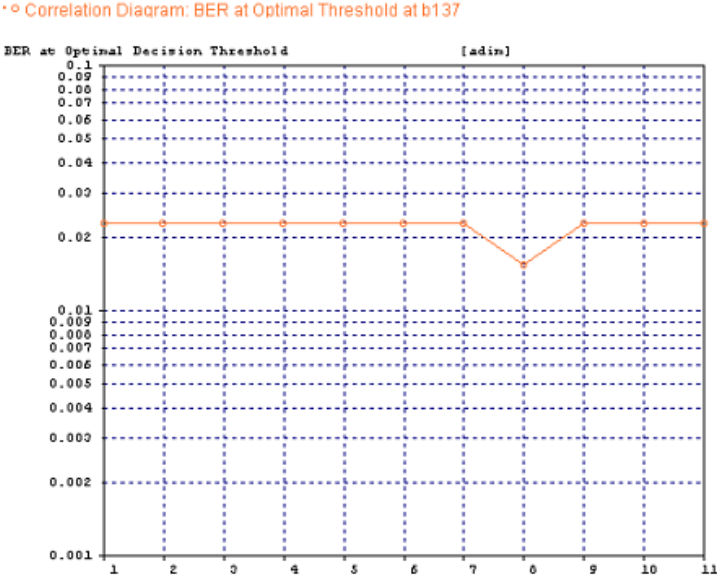

(b)

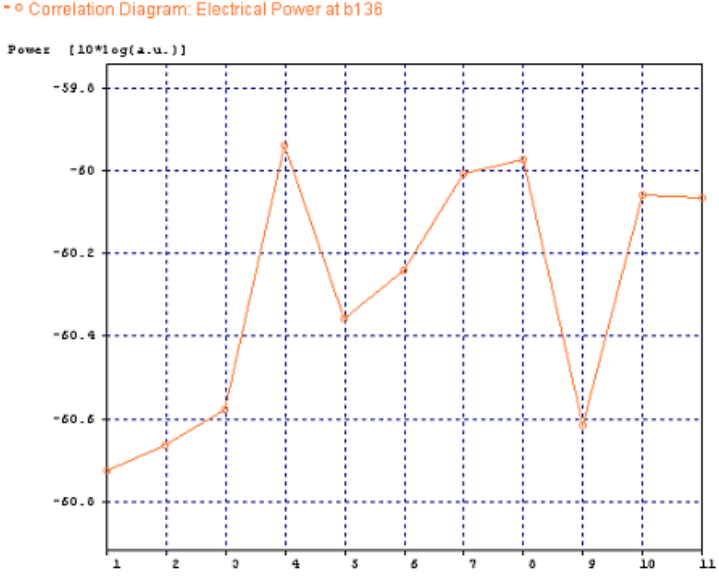

(c)

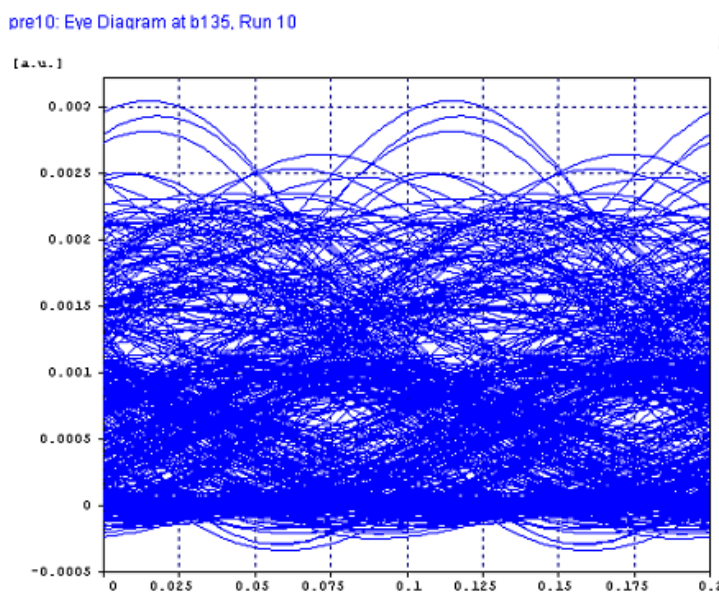

(d)

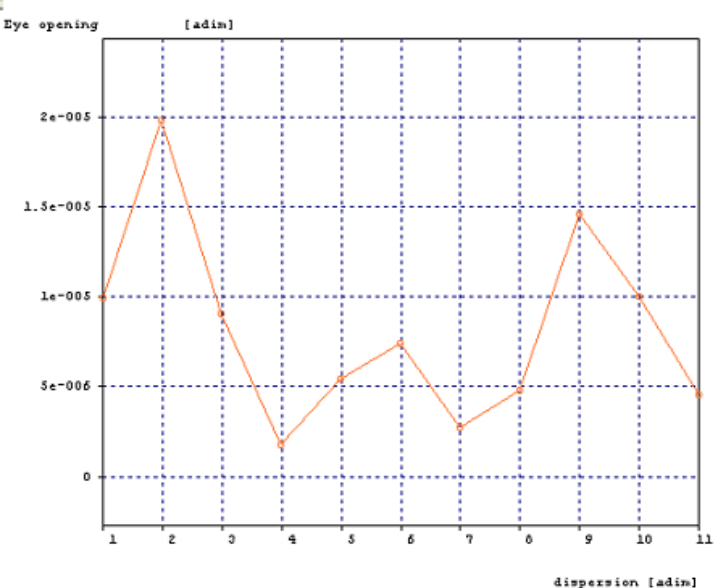

(e)

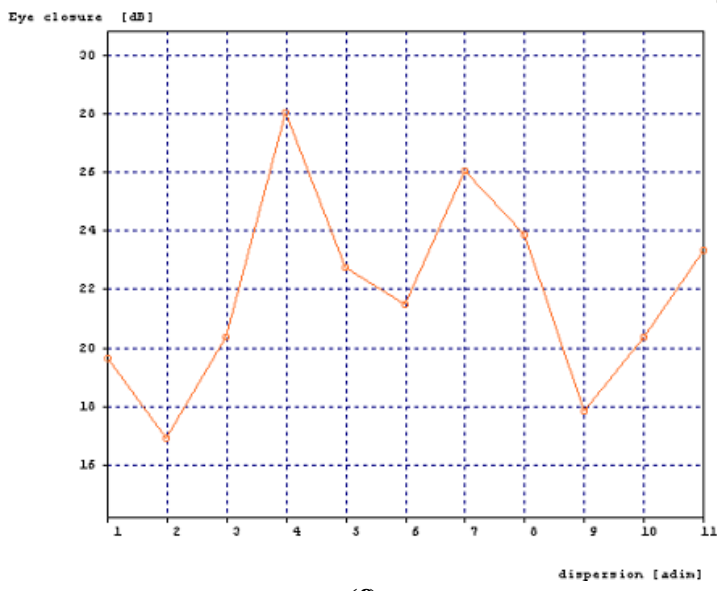

(f)

Figure 1.5: For channel 1 at $10 \mathrm{~Gb} / \mathrm{s}$ for NRZ raised cosine (a) PMD verses equivalent $Q$ factor (b) PMD verses BER (c) the PMD verses electrical power (d) eye diagram at $10 \mathrm{ps} / \sqrt{k m}$ (e) PMD verses eye opening (f) PMD verses eye closure. 


\section{RESULTS FOR POST COMPENSAT-ION}

Now we show the effect of the post compensation technique on the first order PMD. The effect of PMD at different bit rates is shown below :

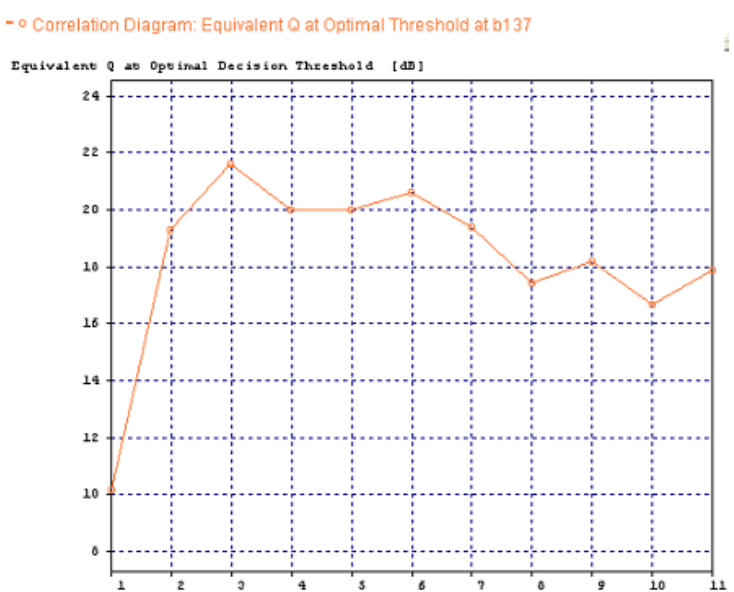

(a)

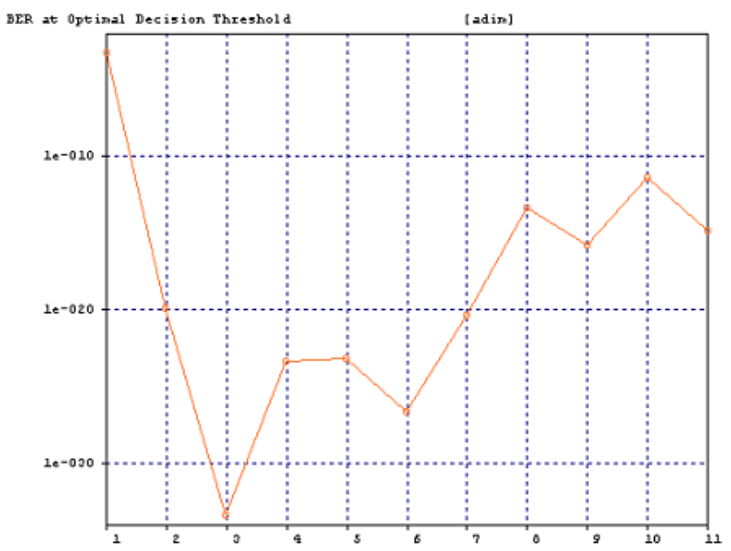

(b)

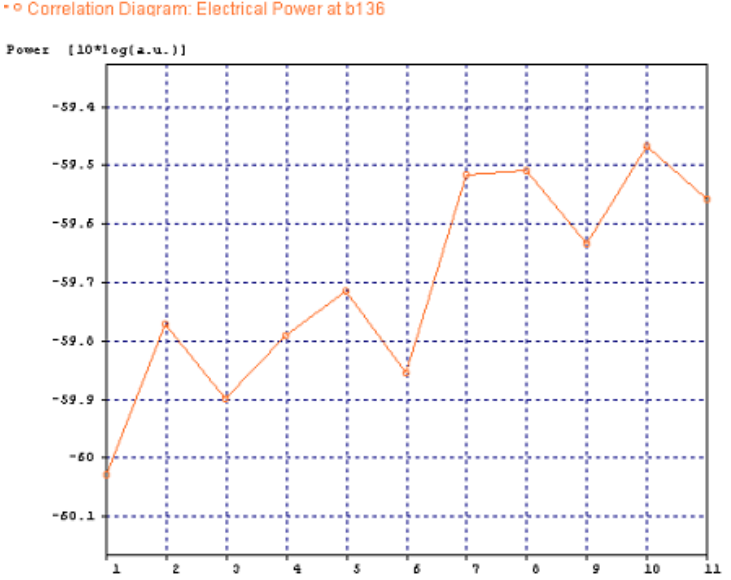

(c)

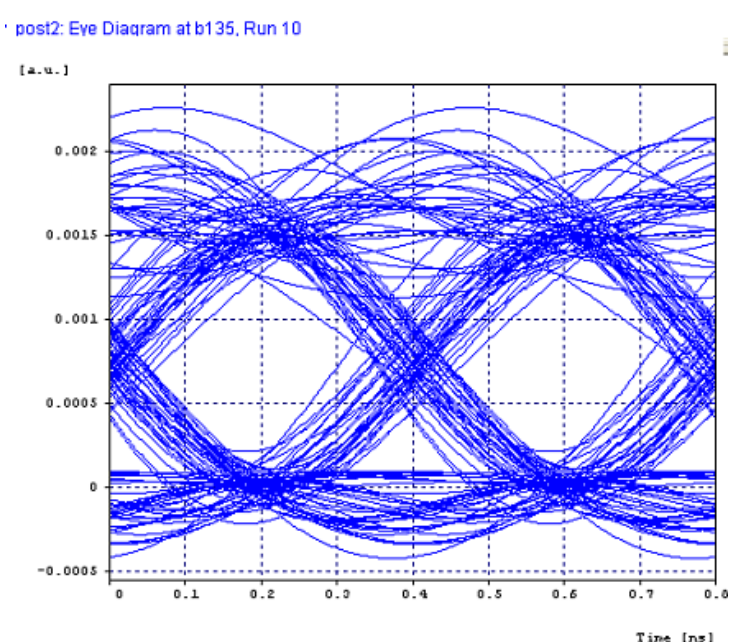

(d)

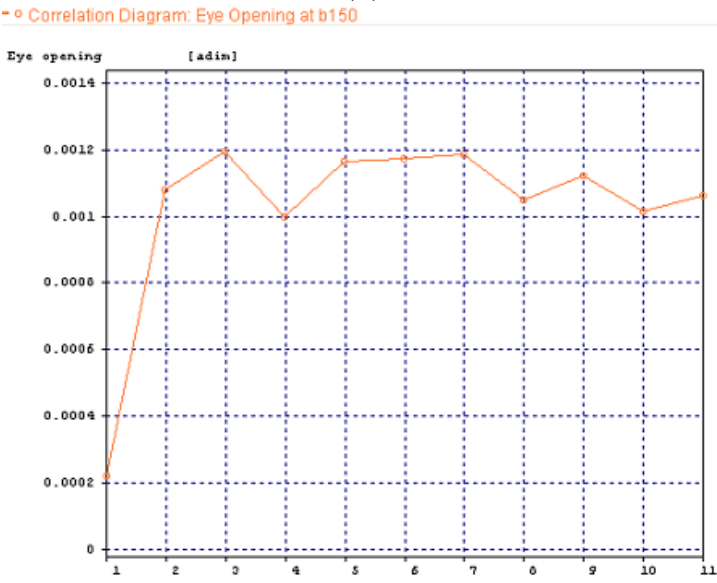

(e)

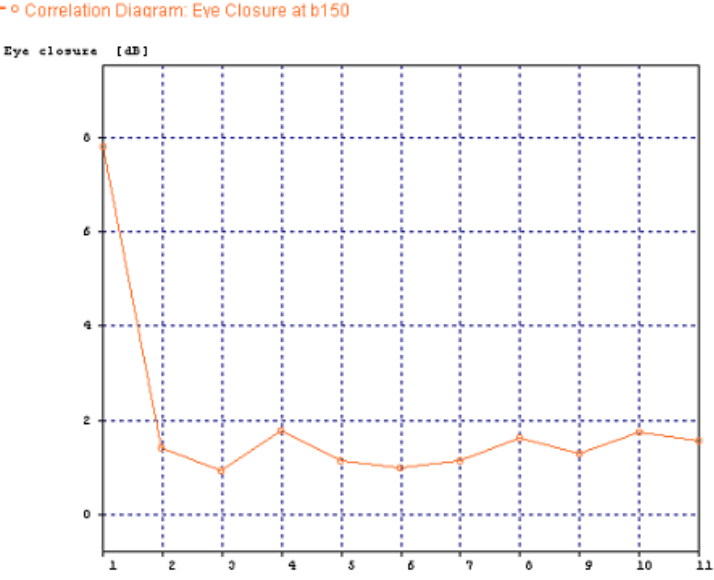

(f)

Figure 1.6: For channel 1 at $2.5 \mathrm{~Gb} / \mathrm{s}$ for NRZ raised cosine (a) PMD verses equivalent $Q$ factor (b) PMD verses BER (c) the PMD verses electrical power (d) eye diagram at $10 \mathrm{ps} / \sqrt{\mathrm{km}}$ (e) PMD verses eye opening (f) PMD verses eye closure. 
The figure 1.7 shows the Q value, BER, electrical power, eye opening, eye closure and eye diagrams at the corresponding values of PMD. All these figures are for the channel one of the transmitter section at $5 \mathrm{~Gb} / \mathrm{s}$.

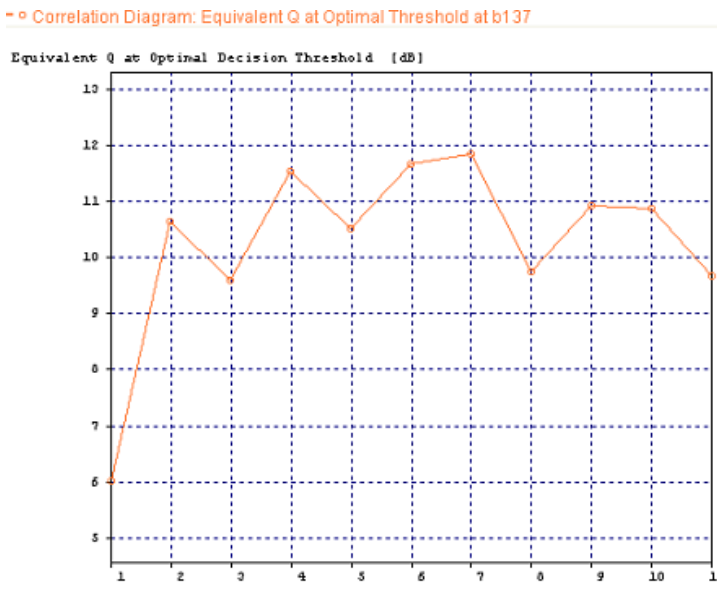

(a)

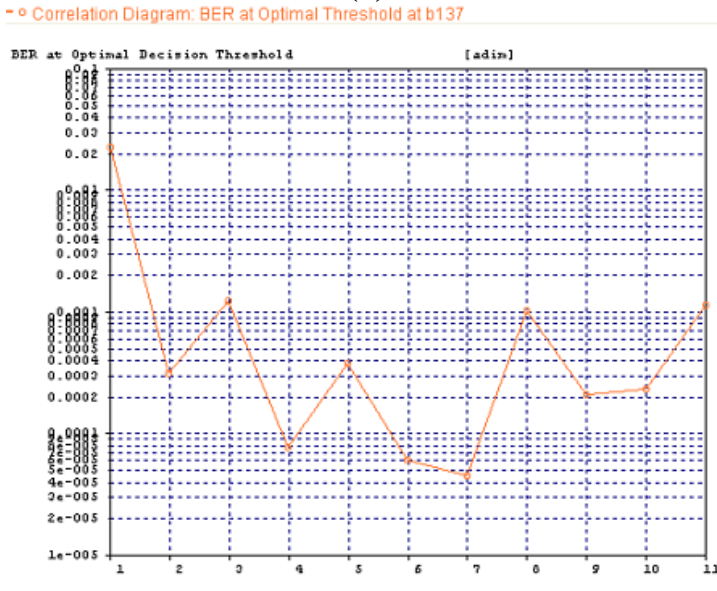

(b)

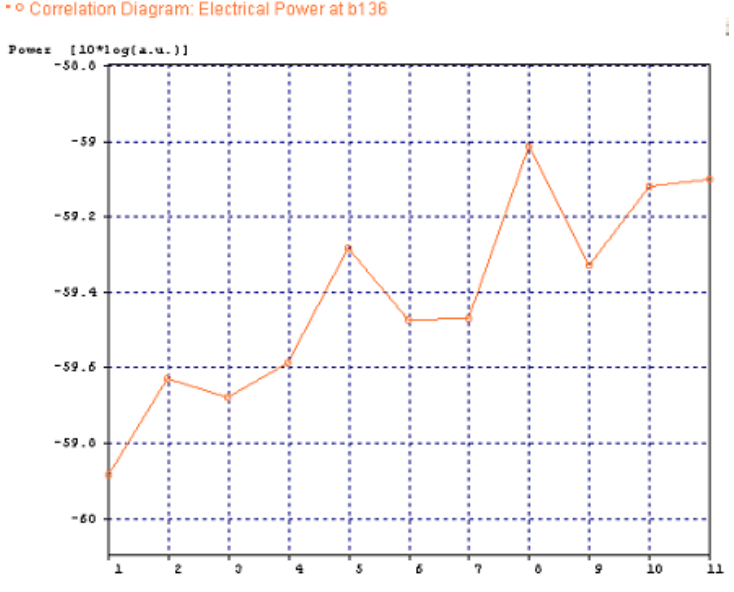

(c)

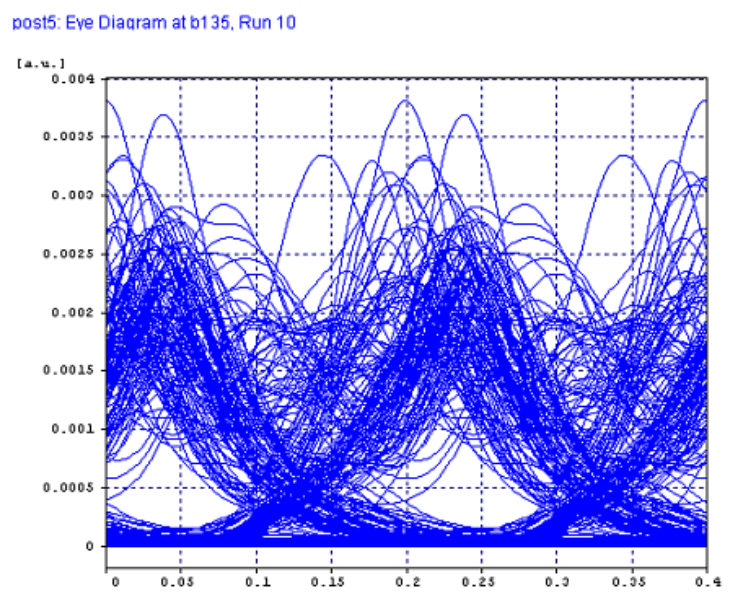

(d)

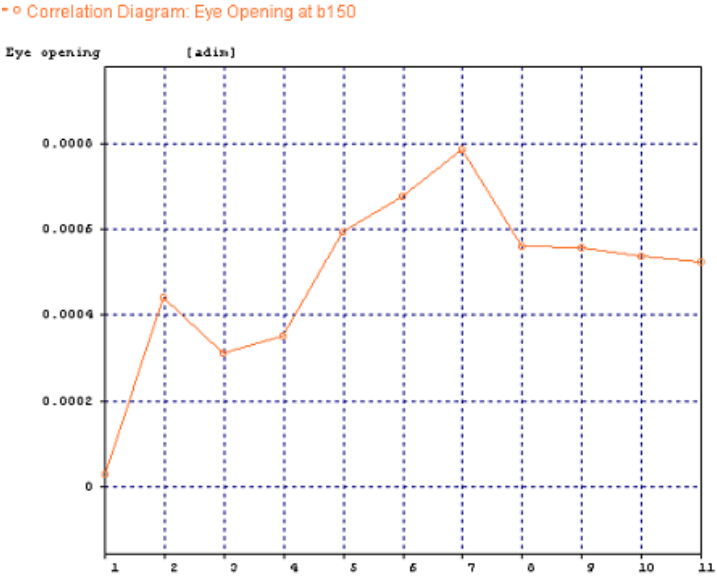

(e)

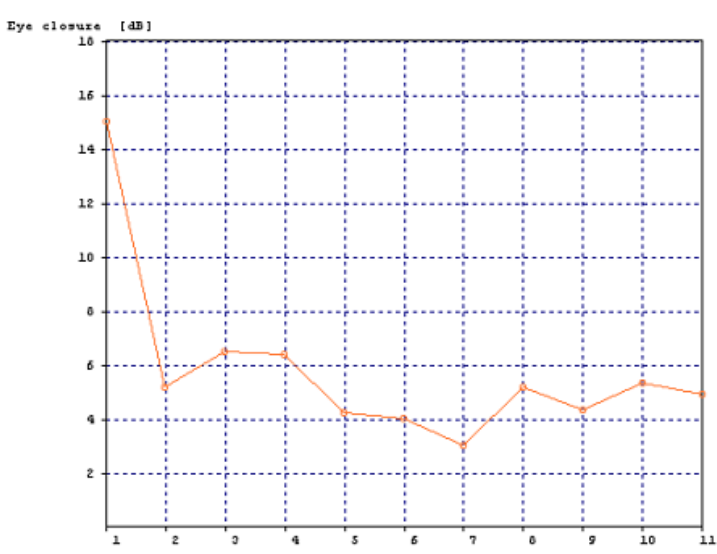

(f)

Figure 1.7: For channel 1 at $5 \mathrm{~Gb} / \mathrm{s}$ for NRZ raised cosine (a) PMD verses equivalent $Q$ factor (b) PMD verses BER (c) the PMD verses electrical power (d) eye diagram at $10 \mathrm{ps} / \sqrt{\mathrm{km}}$ (e) PMD verses eye opening (f) PMD verses eye closure. 
Study the Effects and Compensation of Polarization Mode Dispersion (PMD at Different Data Rate

The figure 1.8 shows the $\mathrm{Q}$ value, BER, electrical power, eye opening, eye closure and eye diagrams at the corresponding values of PMD. All these figures are for the channel one of the transmitter section at $10 \mathrm{~Gb} / \mathrm{s}$.

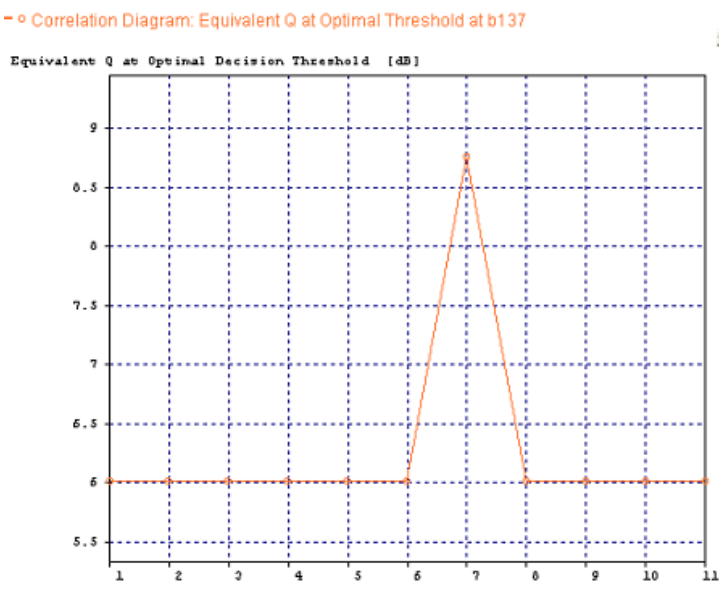

(a)

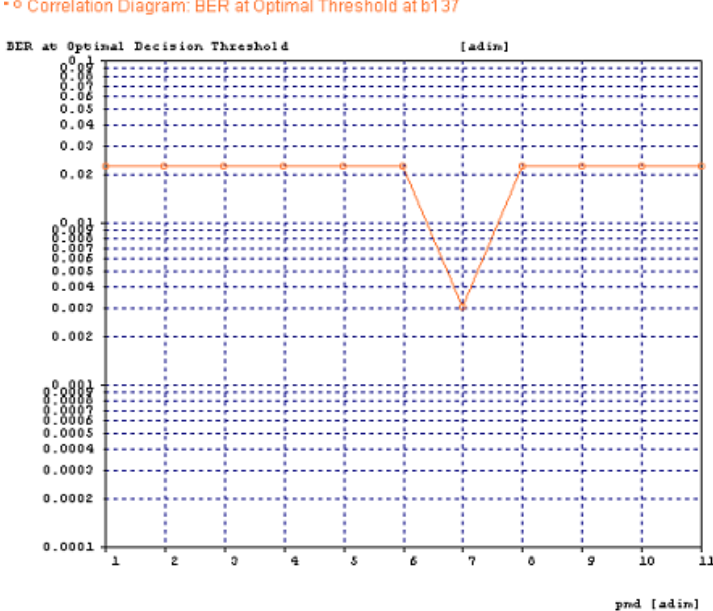

(b)

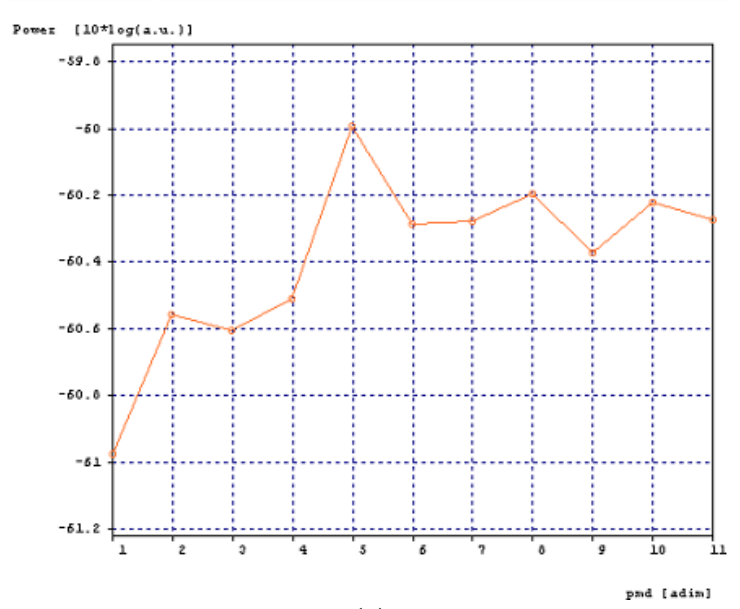

(c)

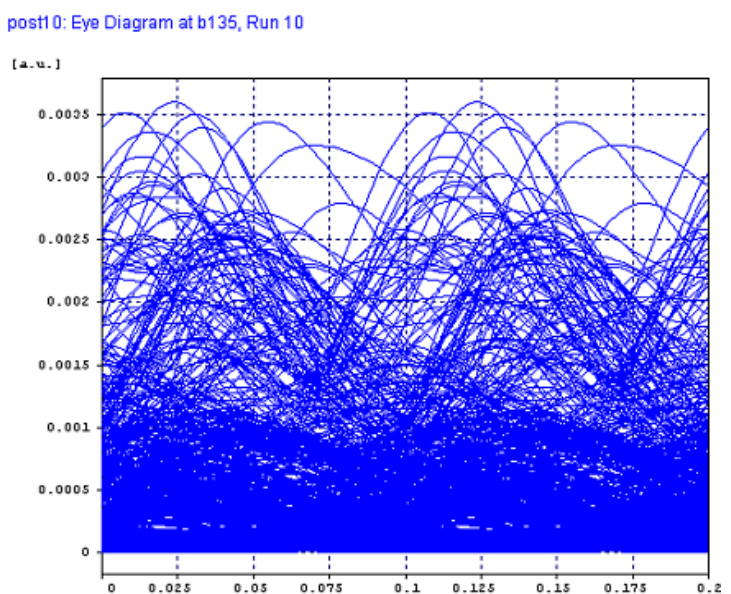

(d)

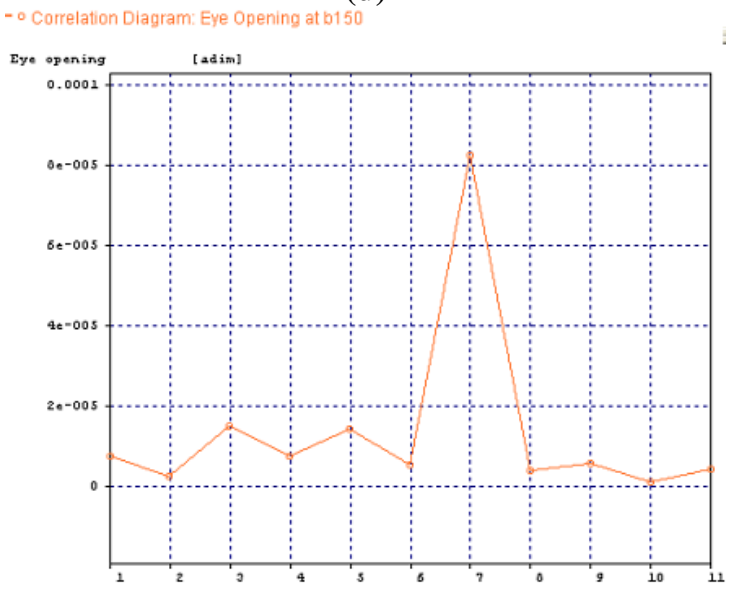

(e)

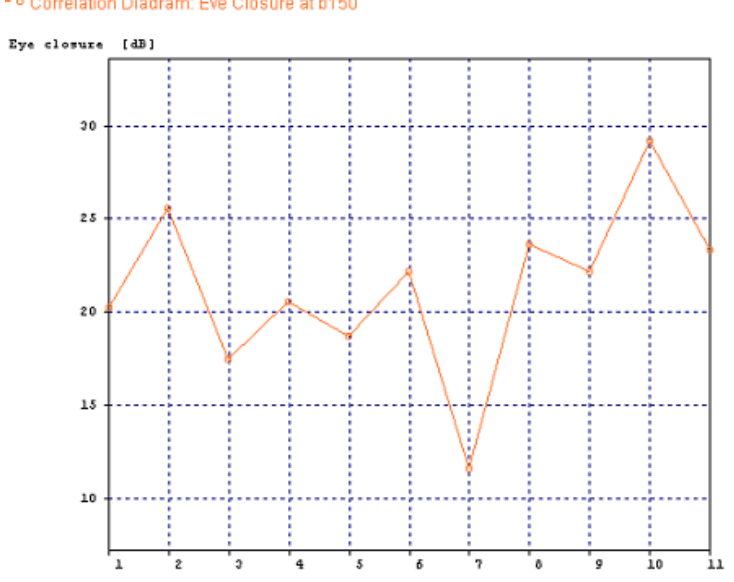

(f)

Figure 1.8: For channel 1 at $10 \mathrm{~Gb} / \mathrm{s}$ for NRZ raised cosine (a) PMD verses equivalent $Q$ factor (b) PMD verses BER (c) the PMD verses electrical power (d) eye diagram at $10 \mathrm{ps} / \sqrt{\mathrm{km}}$ (e) PMD verses eye opening (f) PMD verses eye closure. 


\section{CONCLUSION}

Form the above results we have shown the variation of BER, Equivalent Q, Electrical power, eye opening, eye closure and eye diagrams at $10 \mathrm{ps} / \sqrt{\mathrm{km}}$. We have shown all these parameters at different bit rates from $2.5 \mathrm{~Gb} / \mathrm{s}$ to $10 \mathrm{~Gb} / \mathrm{s}$. Here, we find the effects of PMD on the output power and the eye diagrams. We find that as the PMD of the fiber channel increases the eye becomes closed. At 0 PMD the eye is more open as compared to the $10 \mathrm{ps} / \sqrt{\mathrm{km}}$. We know that as the PMD increases the BER increases and the output electrical power decreases but as we see in the above figures sometimes the BER decreases and the electrical power increases, this is due to the effect of the dispersion on the PMD. A linear effect of the dispersion compensates the nonlinear effects of the PMD of the fiber. We analyze the effect of the PMD on the system. As the PMD increases the BER of the system increases because the input SOP does not match the PSP at the output of the system. We have also analyzed the effect of PMD on the Q factor of the system. As the PMD of the fiber increases the $\mathrm{Q}$ factor starts decaying.

\section{REFRENCES}

[1] J.H. Winters and M.A. Santoro, “ Experimental equalization of polarization dispersion,” IEEE Photon. Technol. Lett. , vol. 2, pp. 591593, 1990.

[2] T. Ono, S. Yamazaki, H. Shimizu and H. Emura, "Polarization control method for suppressing polarization mode dispersion in optical transmission systems,” J. Lightwave Technol., vol. 12, pp. 891-898, 1994.

[3] T. Takahashi, T.Imai and M. Aiki, "Automatic compensation technique for timewise fluctuation PMD in inline amplifier systems", Electron. Lett. , vol. 30, pp. 348-349, 1994

[4] B. Bakshi, J. Hansryd, P. A. Anderekson, J Brentel, E. Koltveit, B. E. Olsson and M. Karlsson, “ Experimental observation of soliton robustness to polarization mode dispersion pulse broadening”, Electron. Lett., vol. 2, pp. 591-592, 1999.

[5] B. W. Hakki, "Polarization mode dispersion compensation using phase diversity detection", IEEE Photon. Technol. Lett., vol. 9, pp. 121-123, 1997.

[6] J. P. Gordon and H. Kogelnik, "PMD fundamentals: Polarization mode dispersion in optical fibers", vol. 97, pp. 4541-4550, 2000 